\title{
Research on HILS Technology Applied on Aircraft Electric Braking System
}

\author{
Suying Zhou, Hui Lin, and Bingqiang Li \\ School of Automation, Northwest Polytechnical University, Xi'an, China \\ Correspondence should be addressed to Suying Zhou; nwpususu@nwpu.edu.cn
}

Received 2 June 2017; Revised 31 July 2017; Accepted 6 August 2017; Published 11 October 2017

Academic Editor: Ephraim Suhir

Copyright ( 2017 Suying Zhou et al. This is an open access article distributed under the Creative Commons Attribution License, which permits unrestricted use, distribution, and reproduction in any medium, provided the original work is properly cited.

\begin{abstract}
On the basis of analyzing the real-time feature of hardware-in-the-loop simulation of aircraft braking system, a new simulation method based on MATLAB/RTW (Real-Time Workshop) and DSP is introduced. The purpose of this research is to develop a digital control unit with antilock brake system control algorithm for aircraft braking system using HILS. DSP is used as simulator. Using this method, a detailed mathematical modeling of system is proposed first. Studies on reducing sampling time with model simplification and modeling for applying to I/O interface of DSP and HILS are conducted. Compared with other methods, this method is low cost and convenient to implement. By using these methods, we can complete HIL simulation of aircraft braking under various experimental conditions, modify its control laws, and test its braking performance. The results have demonstrated that this platform has high reliability. The algorithm is verified by real-time closed loop test with HILS system and the results are presented.
\end{abstract}

\section{Introduction}

The Hardware In-the-Loop Simulation (HILS) is the technique used in the development and testing of complex and costly systems such as military tactical missiles, aircraft flight control system, satellite control systems, and automotive systems $[1,2]$. It is a type of real-time simulation. HILS is an effective tool for design, performance evaluation, and test of vehicle subsystems such as antilock brake system (ABS), active suspension system, and steering system.

The HIL simulation uses model to simulate the parts which are not available or not easy to test, and the hardware of other parts can be connected to the system directly. HILS has the higher fidelity, so it is often used to verify the correctness and feasibility of the control system scheme. Here, the aim of HIL simulation is to verify the performance of electric braking system, so braking system is hardware, and the airframe of the aircraft is simulated by simulation software.

The HILS system consists of the hardware, software, and interface parts. The software part includes a virtual aircraft dynamic model and the hardware part includes the actual brake system including electromechanical actuator (EMA) and antibraking controller. Two parts are linked by the interface part in a real-time simulation environment.

By using HILS system, we can accomplish performance test and assessment of electric braking system. The control algorithm of braking system can be verified by real-time closed loop test. In the meantime, some certain faults appearing in the practical tests can also be simulated, so that the appropriate solutions and control algorithm can be given.

\section{Composition of Aircraft Braking System}

The aircraft braking system consists of aircraft frame, wheels, landing gears, runway, electric brake controller, motor drive controller, electromechanical actuator (EMA), sensor, and so on. In HILS system, the motor drive controller, electromechanical actuator, electric brake controller, and pressure sensor are the actual objects. The aircraft airframe, wheels, landing gears, runway, and speed sensors of aircraft are replaced by Simulink models. Its structure is shown in Figure 1. 


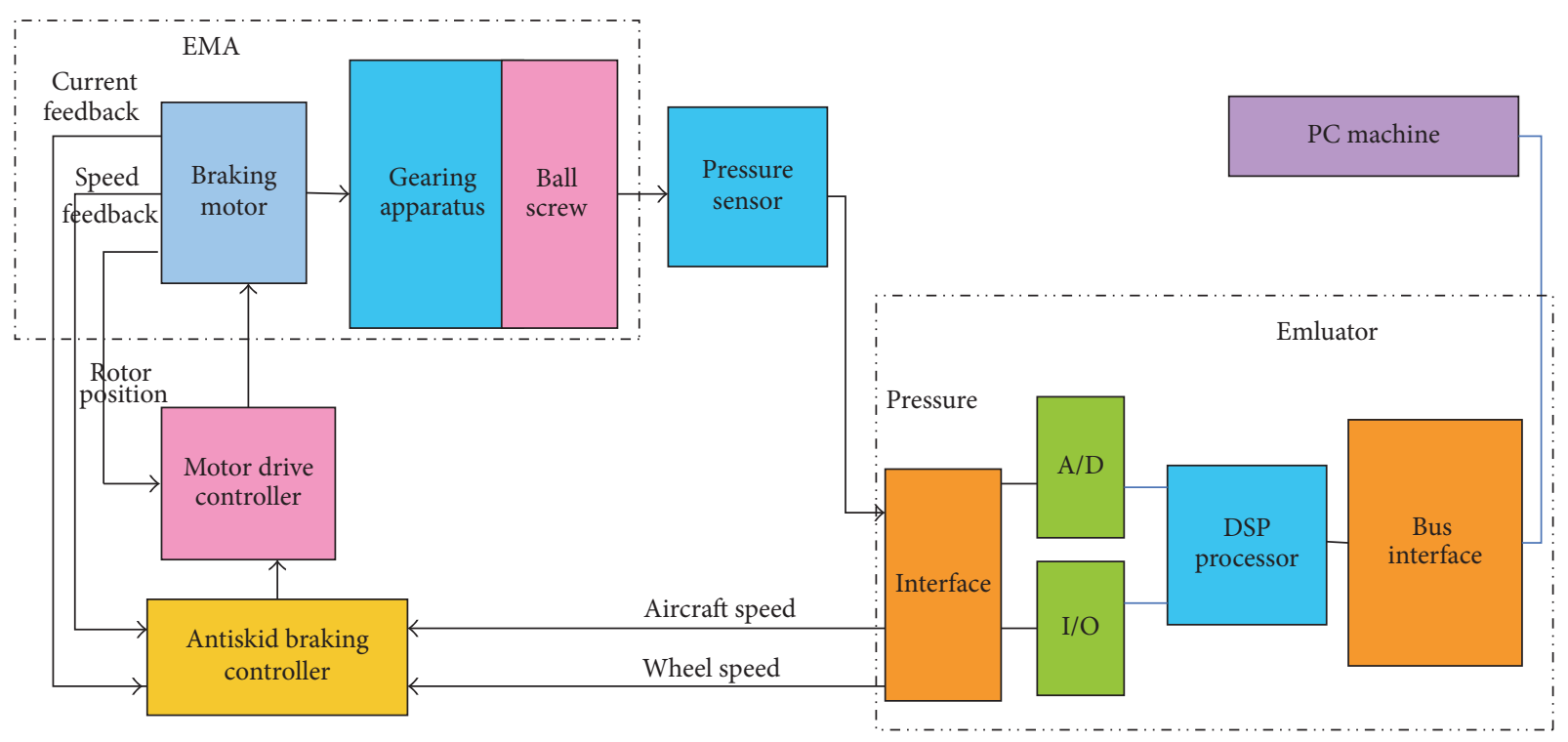

FIGURE 1: The structure of aircraft braking system.

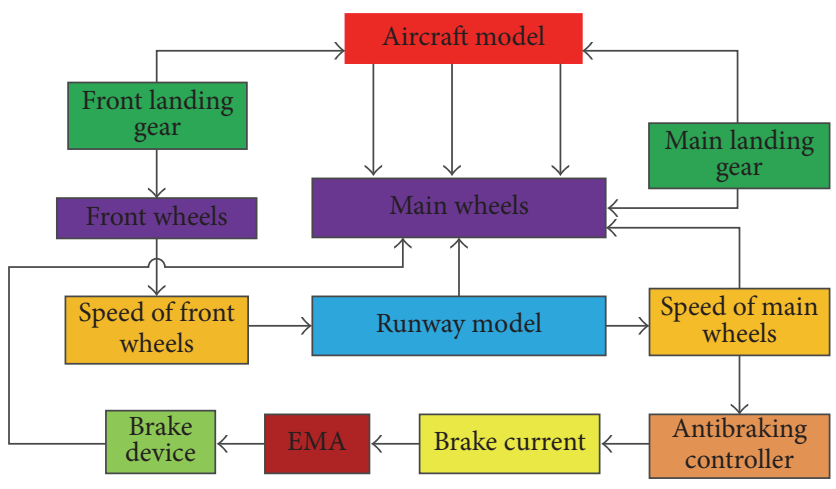

FIGURE 2: The functional relationship between the various parts.

In Figure 1, we are able to understand the working process of electric brake system clearly. We can obtain critical physical quantities, such as the braking distance, aircraft speed, wheel speed, and pitch angles of the aircraft according to the aircraft model. Based on the speed information obtained, braking control controller can calculate the corresponding braking current. Then, the motor generates the corresponding brake pressure, so as to realize the brake of the airplane.

In this paper, the developed all electric braking system model is uploaded and applied to brake experiment. Various brake control law is evaluated by controlling brake system according to different braking conditions.

\section{Dynamic Simulink Model}

According to the functional relationship between the various parts of the brake system, the system structure diagram is shown as in Figure 2.

The aircraft dynamics model is very important for evaluating the performance of antibraking controller, because the accuracy of dynamics model determines the performance of antibraking controller.

Here, we consider the landing gear and the wheels as the part of the airframe and give the main force analysis of the aircraft in the following.

3.1. Airframe Model. The main purpose here is to verify the brake control law by using HILS. The model is based on the following assumptions: first, the aircraft is considered as an ideal rigid body, without considering the elastic deformation. Second, only tackling the longitudinal, vertical, and pitching motion of the aircraft and the tire compression is not considered. The landing gear is regarded as a spring system. The wheel mechanism is consistent and synchronous control [3-6].

The force analysis of airframe is shown in Figure 3.

In Figure 3, $V$ is the speed of the aircraft along the $x$-axis; the unit of $V$ is $\mathrm{m} / \mathrm{s} . m_{A}$ is the aircraft quality; the unit of $m_{A}$ is $\mathrm{kg} . \mathrm{g}$ is the acceleration of gravity; the unit of $g$ is $\mathrm{m} / \mathrm{s}^{2} . F_{R}$ is the engine thrust; the unit of $F_{R}$ is N. $Q$ is the pneumatic 


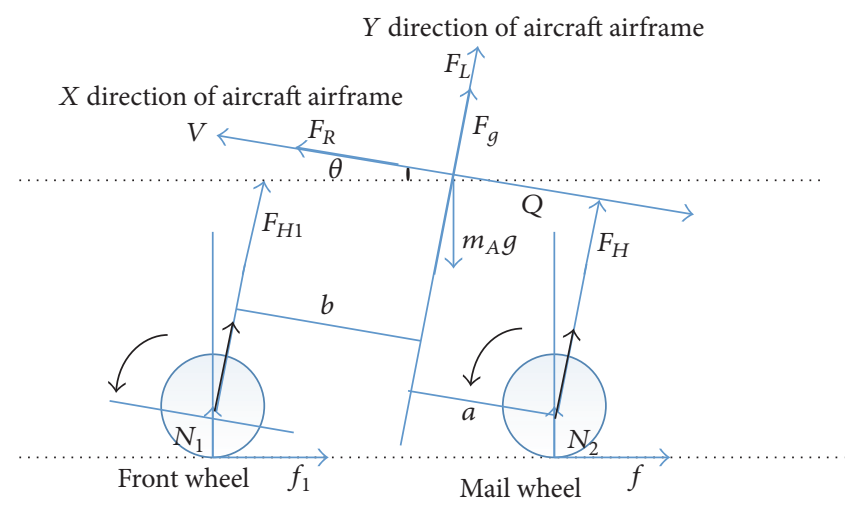

FIgURE 3: The force analysis of airframe.

resistance; the unit of $Q$ is $N . f_{1}$ is the binding force between front wheel and runway; the unit of $f_{1}$ is N. $f$ is the binding force between main wheel and runway; the unit of $f$ is N. $F_{L}$ is aircraft lift; the unit of $F_{L}$ is N. $F_{g}$ is the ground effect force of the airframe when aircraft touches the ground; the unit of $F_{g}$ is N. $F_{H 1}$ is buffer support force of front landing gear; the unit of $F_{H 1}$ is N. $F_{H}$ is buffer support force of main landing gear; the unit of $F_{H}$ is N. $N_{1}$ is front wheel load; the unit of $N_{1}$ is N. $N_{2}$ is main wheel load; the unit of $N_{2}$ is N. $b$ is the horizontal distance from the front wheel to the center of gravity; the unit of $b$ is $\mathrm{m}$. $a$ is the horizontal distance from the main wheel to the aircraft center of gravity; the unit of $a$ is $\mathrm{m}$.

Considering the analysis of the force of the aircraft, according to Newton's second law, the balance equation of the aircraft can be obtained:

$$
\begin{aligned}
m_{A}\left(a_{X}+W V_{Y}\right) & =F_{R}-f_{1}-n f-m_{A} g \sin \theta-Q, \\
m_{A}\left(a_{Y}-W V_{X}\right) & =F_{L}+F_{H 1}+n F_{H}+F_{g}-m_{A} g \cos \theta, \\
Q & =\frac{1}{2} C_{d} \rho_{a} S_{A} V^{2}, \\
F_{L} & =\frac{1}{2} C_{L} \rho_{a} S_{A} V^{2} .
\end{aligned}
$$

Here, $n$ is the number of main wheels and $C_{d}$ is the aerodynamic drag coefficient. $\rho_{a}$ is the air density; the unit of $\rho_{a}$ is $\mathrm{kg} \mathrm{sec}^{2} / \mathrm{m}^{4} . S_{A}$ is the wing area; the unit of $S_{A}$ is $\mathrm{m}^{2} . C_{L}$ is the lift coefficient. $V_{X}$ is the course speed of airframe; the unit of $V_{X}$ is $\mathrm{m} / \mathrm{s}$. $V_{Y}$ is the speed perpendicular to the course of the airframe; the unit of $V_{Y}$ is $\mathrm{m} / \mathrm{s} . a_{X}$ is the acceleration of aircraft along the $X$ axis; the unit of $a_{X}$ is $\mathrm{m} / \mathrm{s}^{2} . a_{Y}$ is the acceleration of aircraft along the $y$-axis; the unit of $a_{Y}$ is $\mathrm{m} / \mathrm{s}^{2} . W$ is the pitching angular speed of aircraft; the unit of $W$ is $\mathrm{rad} / \mathrm{s}$.

3.2. Wheels and Runway Models. According to the law of moment of inertia, the force equation of the wheel is listed as the following formula:

$$
f R-T_{b}-B_{w} \omega=\dot{\omega} J_{w}
$$

In formula (2), $R$ is the radius of main wheel; the unit of $R$ is $\mathrm{m} . T_{b}$ is the brake torque; the unit of $T_{b}$ is $\mathrm{N} \cdot \mathrm{m} . B_{w}$ is the axle friction coefficient. $\omega$ is the main wheel angular velocity; the unit of $\omega$ is $\mathrm{rad} / \mathrm{s}$. $J_{w}$ is the wheel inertia; the unit of $J_{w}$ is $\mathrm{kg} \mathrm{sec}{ }^{2} \mathrm{~m}$.

Here, the sliding of the aircraft wheel relative to the ground is defined as the relative slip rate; the formula is as follows:

$$
\lambda=\frac{\left(V_{z x}-R \omega\right)}{V_{z x}} .
$$

In formula (3), $V_{z x}$ is wheel speed of aircraft.

Factor that affects the binding force $f$ is called the friction coefficient. We can define it as the following formula:

$$
\mu=\frac{f}{N_{2}} .
$$

3.3. Design of Autobrake Control Law. Automatic brake system refers to the brake system that is slowed by electric brake to prevent the aircraft from flowing out of the runway. Automatic brake selector switch is used to select the brake stalls; then the system puts in braking performance according to the constant deceleration rate [7].

After the deceleration rate is selected, the reference speed of the aircraft can be obtained. The reference speed and the actual feedback of the aircraft can be used to adjust the brake current signal through control algorithm.

The control strategy adopted is $\mathrm{PD}+\mathrm{PBM}$ antiskid control combined with a given rate of deceleration tracking control. The brake control part adopts the control scheme of given deceleration rate and $\mathrm{PD}+\mathrm{PBM}$ control law is used in antiskid control. When the antiskid control is acting during braking, the output of the brake system maintains the output of previous moment. The output of control law is the difference between brake current and antiskid current. The realization of the algorithm is shown as in Figure 4.

In order to improve the robustness and adaptive ability of the automatic braking system, a quasi-sliding mode variable structure control based on fuzzy control is designed.

Here, we can define the error of speed as the following formula:

$$
s=\int_{0}^{t} a_{\text {given }}-V_{x} .
$$




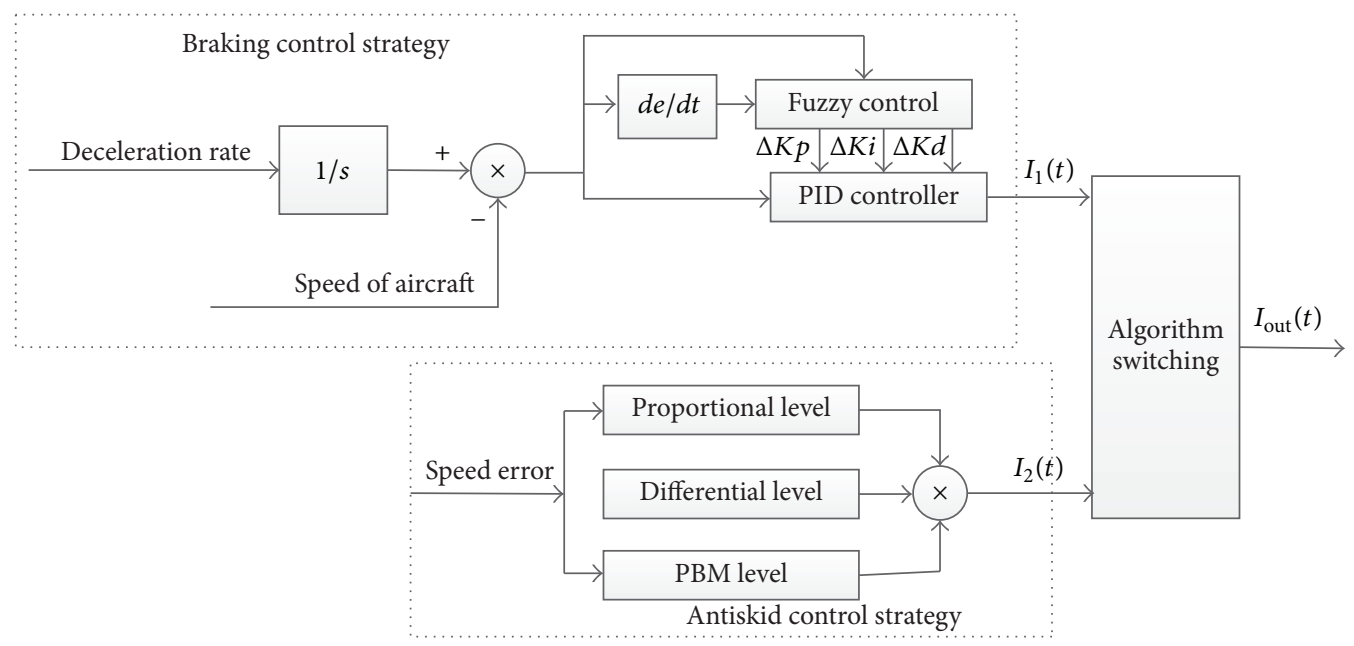

FIGURE 4: The control strategy of braking system.

In formula (5), $a_{\text {given }}$ is deceleration rate.

$$
\begin{aligned}
& I_{1}(t) \\
& = \begin{cases}0, & s>\varepsilon \text { or } s<-\varepsilon, \\
k_{p} \cdot s+k_{i} \int s d t+k_{d} \cdot \frac{d s}{d t}, & -\varepsilon \leq s \leq \varepsilon,\end{cases} \\
& \text { Ibrake }(t)= \begin{cases}\text { const, } & I_{1}(t) \geq \text { given value, } \\
I_{1}(t), & \text { others, } \\
0, & I_{1}(t)<0,\end{cases} \\
& I_{\text {out }}= \begin{cases}I \text { brake, } & \text { if } I_{2} \leq \zeta, \\
\text { Ibrake } \text { br-1 }_{k}-I_{2}, & \text { if } I_{2}>\zeta .\end{cases}
\end{aligned}
$$

Here, $\zeta$ and $\varepsilon$ are very small boundary value. $I_{1}(t)$ is braking current. $k_{p}, k_{i}$, and $k_{d}$ are proportion coefficient, differential coefficient, and integral coefficient, respectively.

And the proportion coefficient $k_{p}$, integral coefficient $k_{i}$, and differential coefficient $k_{d}$ have important influence on the performance of the brake system. In order to improve the robustness and adaptive ability of the automatic braking system, fuzzy control is designed to adjust PID parameters online.

In the part of fuzzy parameter correction, the velocity error and the derivative of the velocity error are the input of the fuzzy controller, and the change of the three parameters of the PID is the output of the fuzzy controller [8].

According to the influence of control parameters on the output characteristics of the system, we can obtain adjustment rules of the parameter. When speed error is large, in order to improve the response speed of the system and prevent the differential overflow caused by the large transient, we should take a larger $k_{p}$ and smaller $k_{d}$.

At the same time, in order to prevent integral saturation and avoid large overshoot, the integral separation PID regulation should be adopted. When the error is medium
TABLE 1: Fuzzy rules of $\Delta k_{p}$.

\begin{tabular}{lccccccc}
\hline e & & & \multicolumn{7}{c}{ ec } & PS & PM & PB \\
\hline NB & PB & PB & PM & PM & PS & Z & Z \\
NM & PB & PB & PM & PS & PS & Z & NS \\
NS & PM & PM & PM & PS & Z & NS & NS \\
Z & PM & PM & PS & Z & NS & NM & NM \\
PS & PS & PS & Z & NS & NS & NM & NM \\
PM & PS & Z & NS & NM & NM & NM & NB \\
PB & Z & Z & NM & NM & NM & NB & NB \\
\hline
\end{tabular}

large, in order to reduce the overshoot of the system response and ensure response speed, the smaller $k_{p}$, smaller $k_{i}$, and moderate size $k_{d}$ should be chosen. When speed error is the lowest, in order to make the system have steady performance and avoid the oscillation of the system in the vicinity of the reference speed, it is necessary to take a larger $k_{p}, k_{i}$, and proper $k_{d}$.

In addition, when $\dot{s}$ is smaller, the differential coefficient $k_{d}$ should be larger. When $\dot{s}$ is larger, the differential coefficient $k_{d}$ should be smaller. According to the regulation rules, the fuzzy rules can be designed and adjusted.

Fuzzy sets of fuzzy parameters are defined as

$$
\begin{aligned}
& s=\{\mathrm{NB} \text { NM NS Z PS PM PB }, \\
& \dot{s}=\{\mathrm{NB} \text { NM NS Z PS PM PB }, \\
& \Delta k_{p}=\{\mathrm{NB} N \mathrm{NM} \text { Z PS PM PB }\}, \\
& \Delta k_{i}=\{\mathrm{NB} \text { NM NS Z PS PM PB }\} \text {, } \\
& \Delta k_{d}=\{\mathrm{NB} \text { NM NS Z PS PM PB }\} .
\end{aligned}
$$

For example, fuzzy rules of $\Delta k_{p}$ can be obtained as in Table 1.

By using this method, the fuzzy rules of other parameters can also be obtained. 


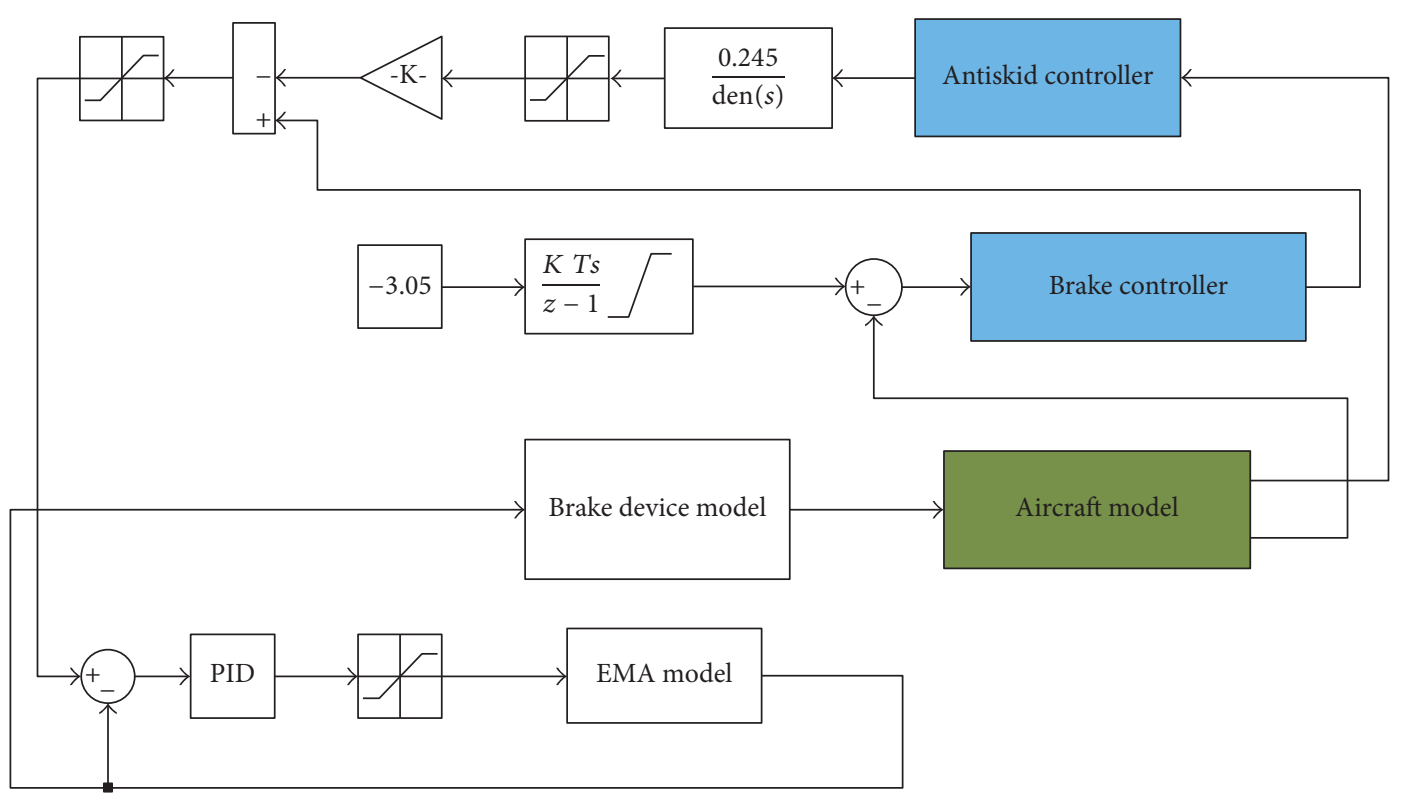

FIGURE 5: The integrated model of electric braking system.

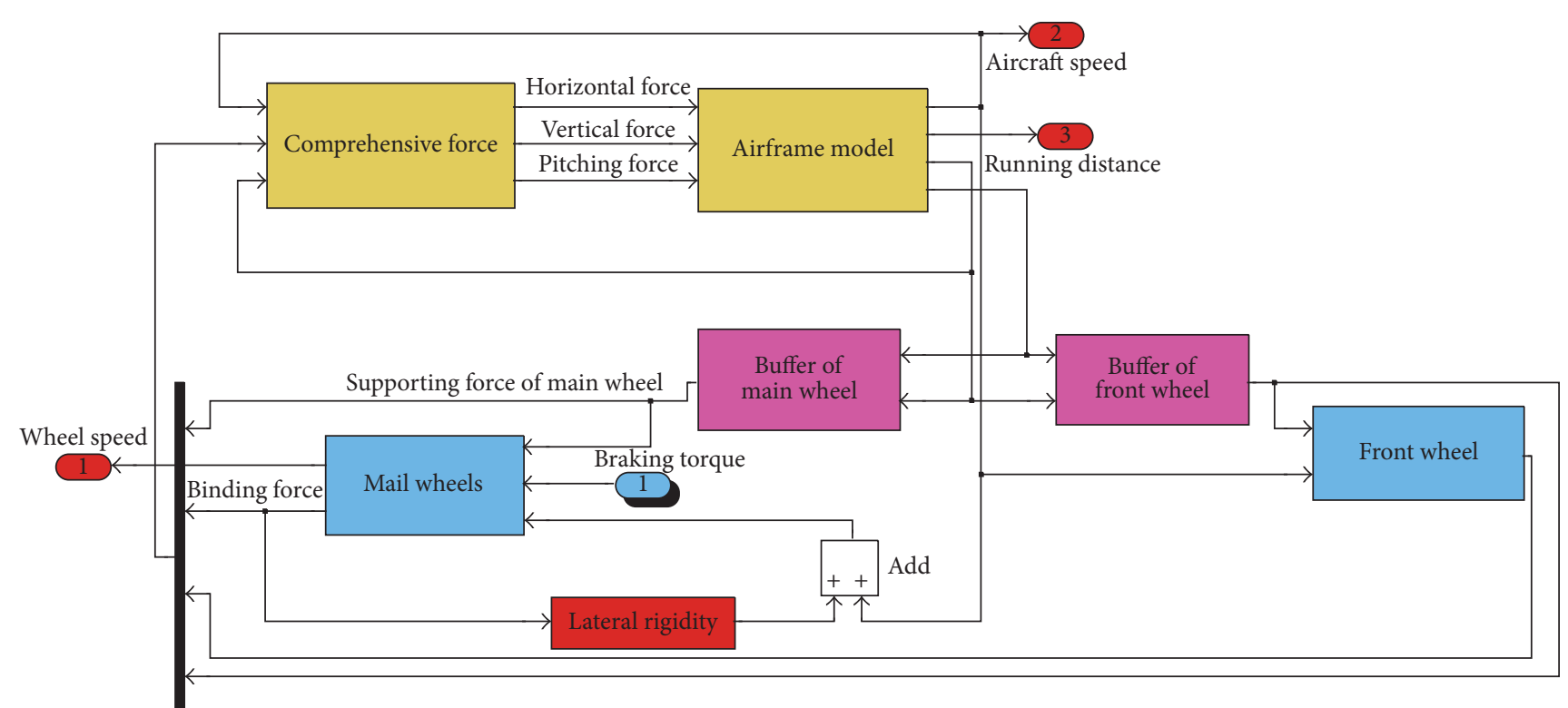

FIGURE 6: The simulation model of airframe.

3.4. Simulink Model of Aircraft Electric Braking System. The establishment of simulation model is one of the key technologies in HILS. The simulation model will be directly related to the authenticity of HILS. Therefore, in order to establish the simulation model of aircraft dynamics in MATLAB and verify the accuracy of the model, we must establish the whole mathematical model of aircraft. Then, landing gear, tires, and other factors must be considered.
According to the mathematical formula given in the previous section, the integrated model of aircraft electric braking system can be built as in Figure 5.

The brake system model mainly consists of airplane model, brake controller model, brake device model, and electromechanical actuator model.

According to the mathematical model of the aircraft, the simulation model of airframe can be built as in Figure 6 . 


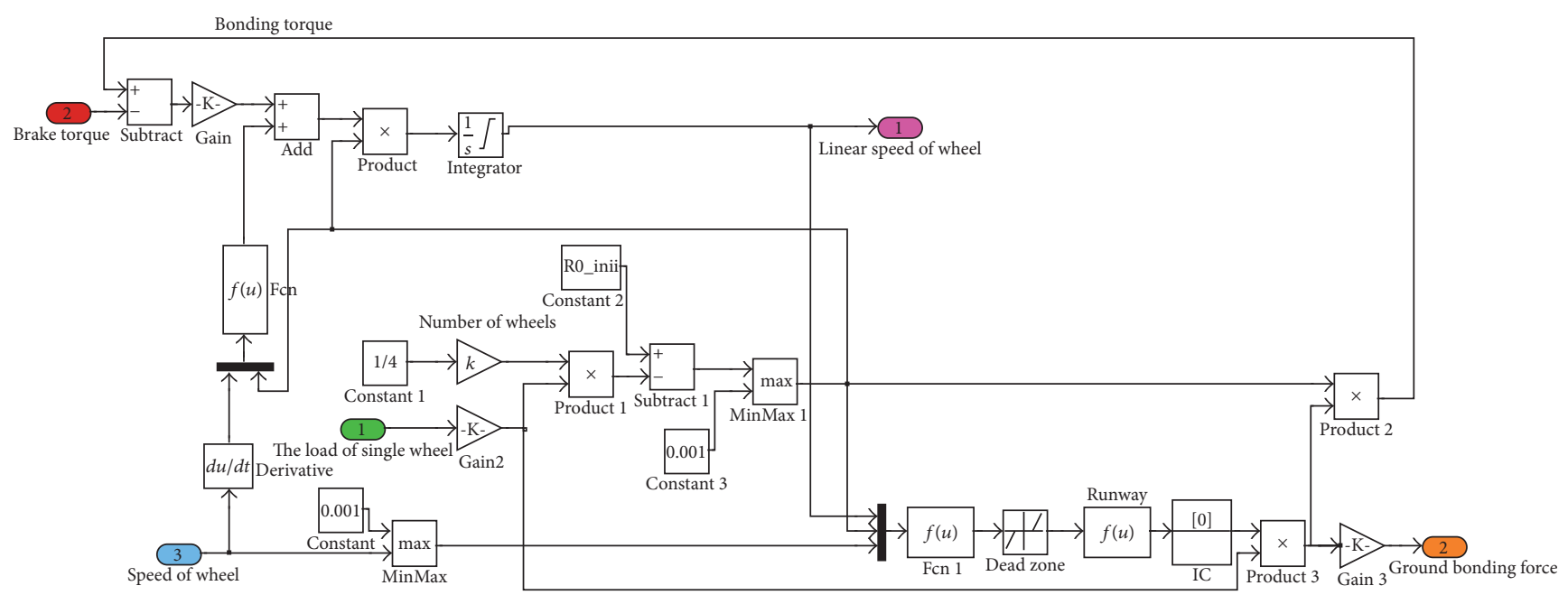

Figure 7: The model of main wheel and runway.

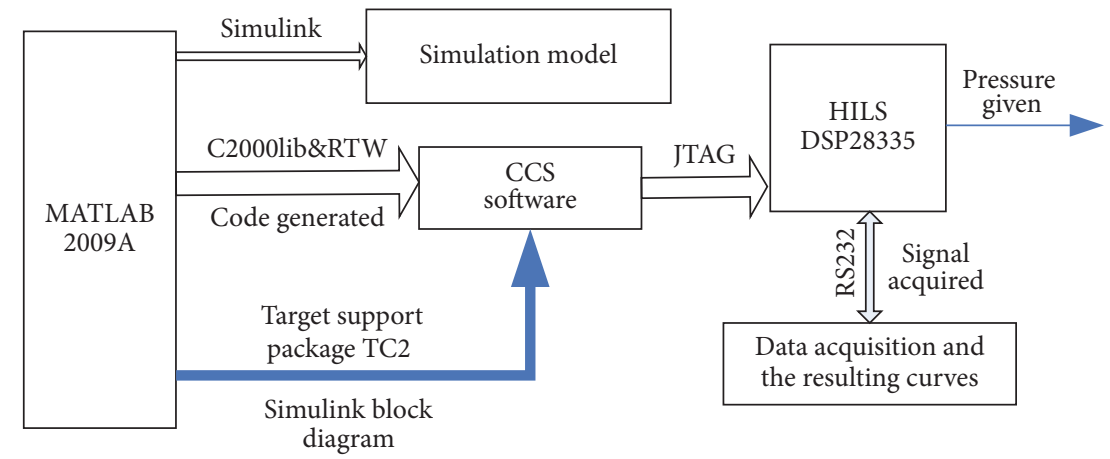

FIGURE 8: The structure of HILS system.

The model of main wheel and runway is shown as Figure 7.

In Figure 7, the brushless DC motor is chosen as the drive motor, and the corresponding mathematical model can be established.

According to the control law designed, the corresponding MATLAB model can be built.

In the same way, we can establish the mathematical model of the other control parts in electric braking system.

\section{Design of All Aircraft Brake HILS System}

The structure of HILS system is shown in Figure 8.

In HILS system, the simulation model should be discretized, and the module that needs to be replaced by the hardware should be determined. After we set the corresponding interface, then the Simulink simulation module can be converted into DSP code through the MATLAB toolbox and CCS software. Here, we call DSP code as digital model of HILS system.
In Figure 8, the simulation model is designed primarily first, just as we have done in the previous part. Then the DSP code can be generated automatically; the experimental results can be uploaded by using the interface part.

In HILS system, the electromechanical actuator and the brake controller are replaced by physical hardware. Other parts are replaced by simulation model.

(1) Simulation Model Discretization and Interface Setting. MATLAB/Simulink provides Target Support Package TC2 software, which supports Taxes Instrument (TI) C2000 series DSP and has various DSP function interface module. By using this tool, we can convert Simulink model of the aircraft braking system into executable code for DSP.

Simulation process based on MATLAB/RTW is listed as follows: an appropriate simulation model is established and discretized in the Simulink environment; then based on RTW simulation platform, real-time code for DSP can be generated and downloaded into the target machine (DSP). By using an external mode, we can adjust simulation parameters online 


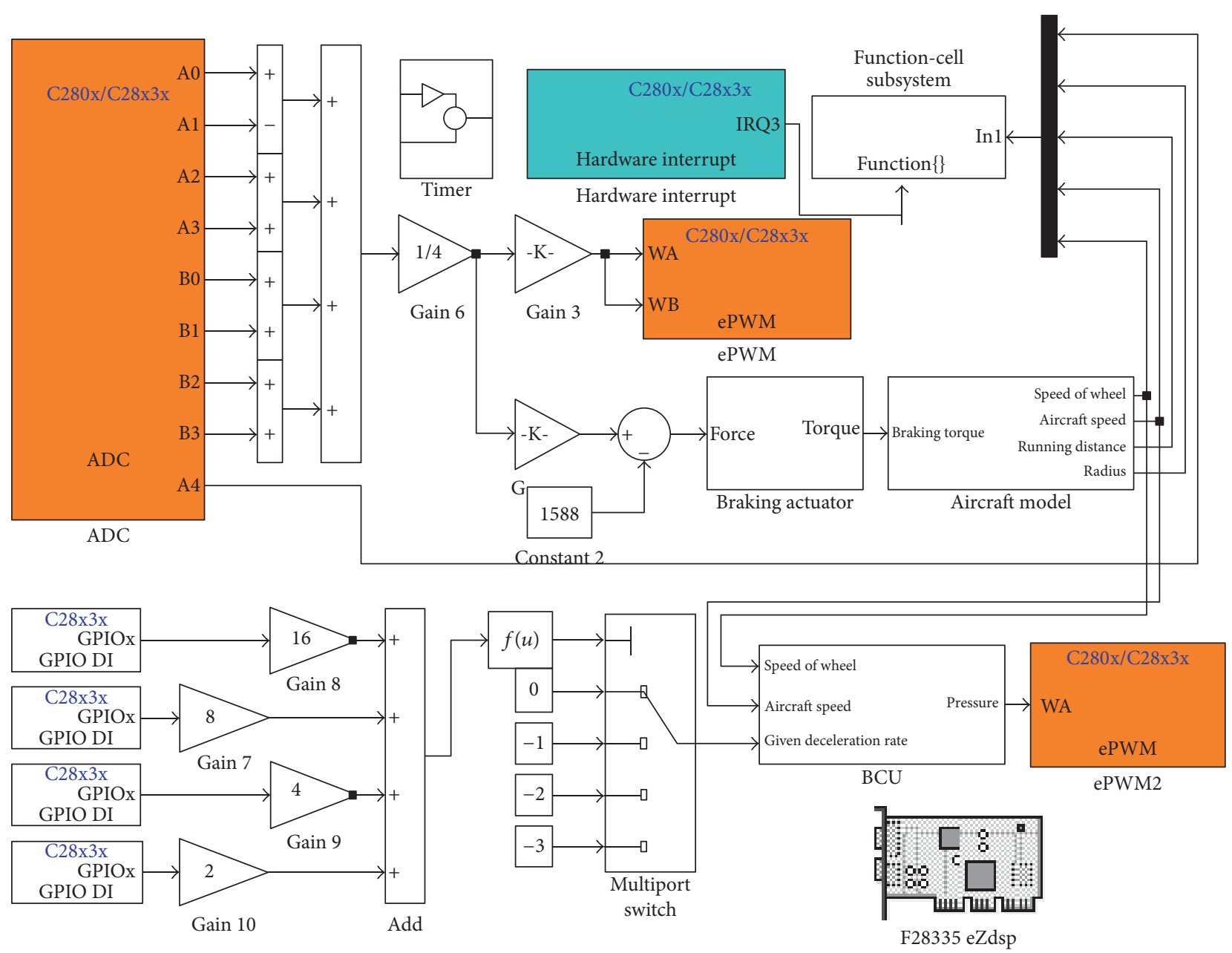

FIGURE 9: The discrete model of braking system.

and modify the simulation model until the results satisfy the design requirements.

In this paper, the discretized mathematical model of aircraft braking system is shown as in Figure 9.

On the basis of building the correct model of electric braking system, the corresponding interface of HILS is designed according to the function block of Target Support Package TC2. In Figure 9, the ADC module has 9 channels. Eight channels are used as pressure acquisition signal of pressure sensor. One channel is used as a trigger signal for PWM.

There are 4 GPIO modules; the position of automatic brake selector switch is determined by 4-bit binary code.

There is one hardware interrupt, SCI module, and timer. Timer is used to generate hardware interrupts. The SCI module uploads the brake signals to the host computer.

(2) The Design of the Hardware. The hardware of HILS system is composed of interface circuit, signal acquisition circuit, signal conditioning circuit, and braking motor. The diagram of the hardware is designed as in Figure 10. Digital signal processing is done by DSP and CPLD.

First, by use of powerful capabilities of DSP, functions such as given pressure regulation, SCI serial communication, brushless DC motor PWM control, the motor position, and speed of capture and signal feedback can be completed.

On the other hand, by using powerful logic computing power of CPLD, functions such as commutation control, enabling control, three phase full-bridge control signal conditioning, and other functions can be implemented.

\section{Test Results}

5.1. Simulation Results. In order to validate the validity of the simulation model and compare with HILS results, here, the performance of electric braking system is simulated under MATLAB/Simulink environment. The simulation studies are carried out when the reduction rate is high and medium, as well as emergency brake.

According to the actual working situation of the aircraft, when deceleration rate of automatic braking is high, the 


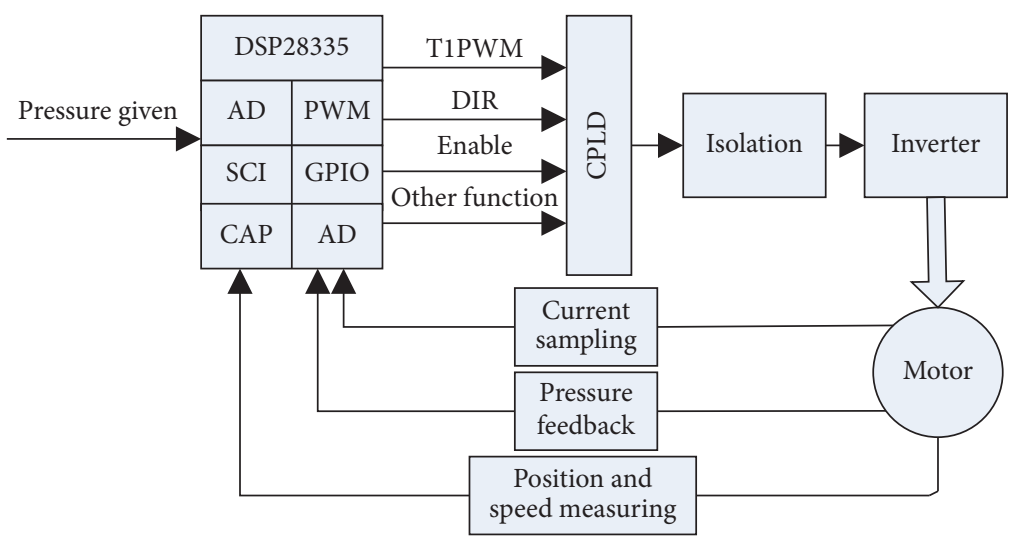

FIgURE 10: The hardware of HILS.

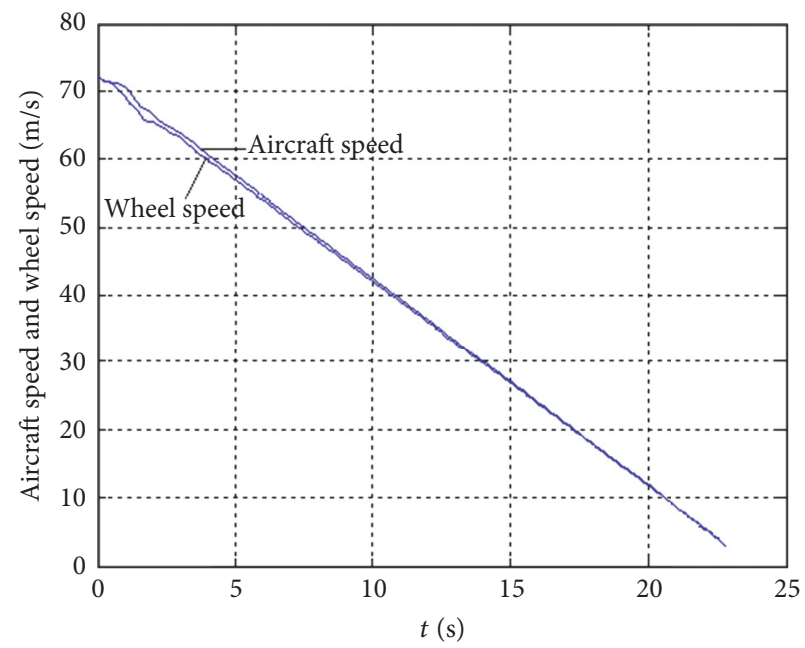

(a) Aircraft speed and wheel speed

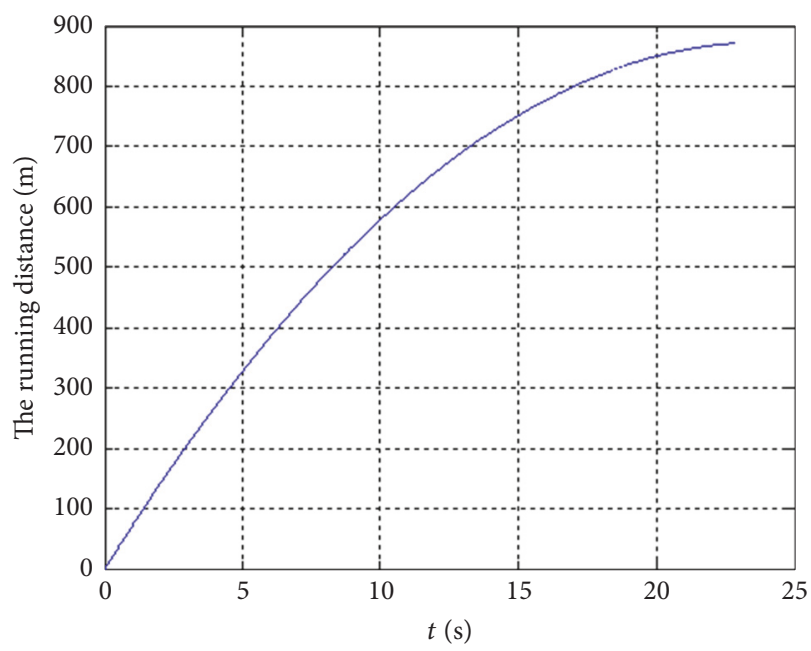

(b) Running distance

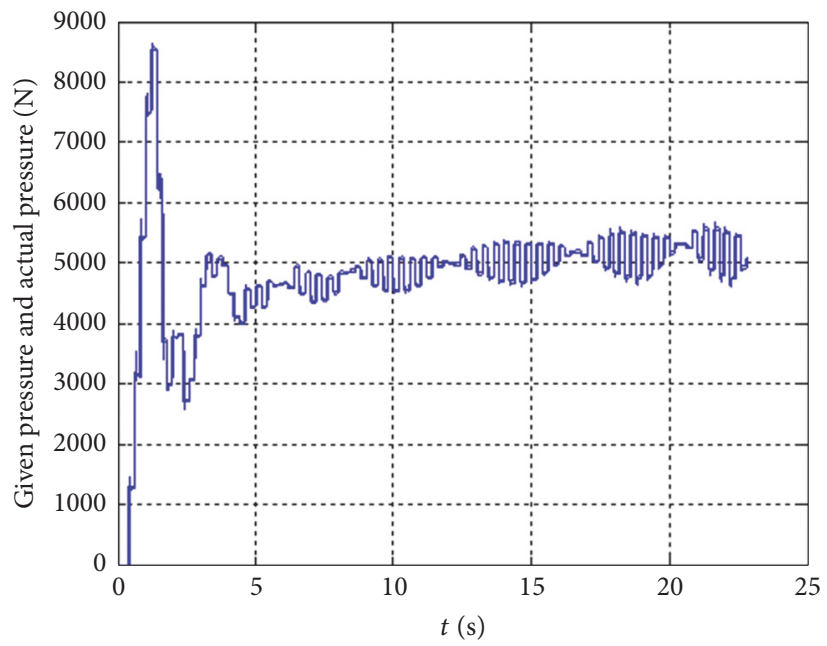

(c) Brake pressure

FIGURE 11: Simulation results when deceleration rate is 3.05. 


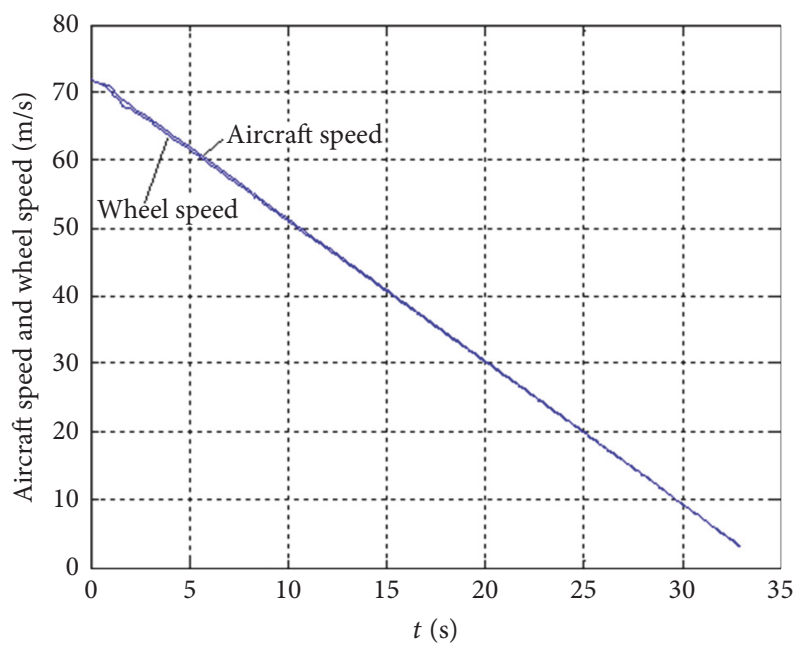

(a) Aircraft speed and wheel speed

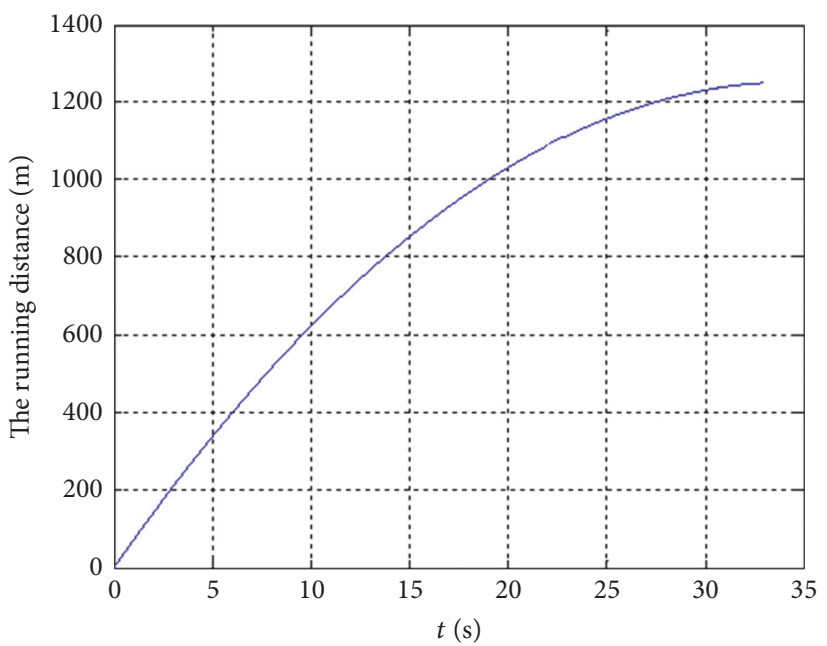

(b) Running distance

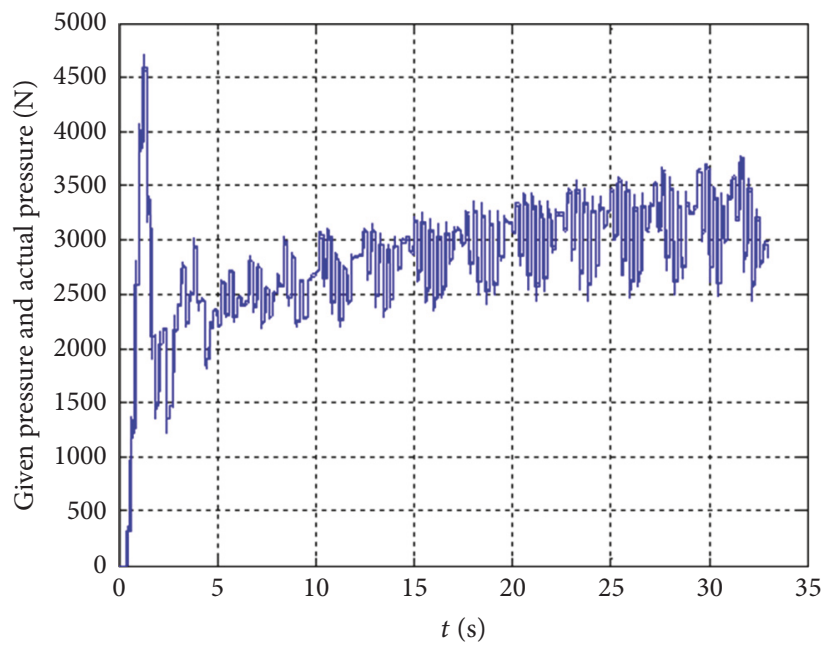

(c) Brake pressure

FIGURE 12: Simulation results when deceleration rate is 2.1.

actual deceleration rate is set to -3.05 , and when the deceleration rate is medium, the actual deceleration rate is set to -2.1 . In the case of emergency braking, the brake is controlled by the maximum braking force. below.

The simulation results of the system model are given

(1) Deceleration Rate Is 3.05. When the deceleration rate is 3.05 , the digital simulation results are shown in Figures 11(a)-11(c).

(2) Deceleration Rate Is 2.1. When the deceleration rate is 2.1, the digital simulation results are shown in Figures 12(a)-12(c).

(3) Emergency Brake. In the case of emergency braking, manual braking mode is used. Maximum braking force is used to achieve emergency brake control.
Here, the digital simulation results are shown in Figures 13(a)-13(c).

5.2. HILS Results. In HILS system, the electromechanical actuator and the brake controller are physical hardware, and other parts are replaced by simulation model.

The experimental study is carried out by using HILS system. In order to compare the results, the simulations when the deceleration rate is 3.05 and 2.1 as well as in case of emergency brake are given. 16.

The experiment results will be given in Figures 14, 15, and

(1) Deceleration Rate Is 3.05. See Figure 14.

(2) Deceleration Rate Is 2.1. See Figure 15.

(3) Emergency Brake. See Figure 16. 


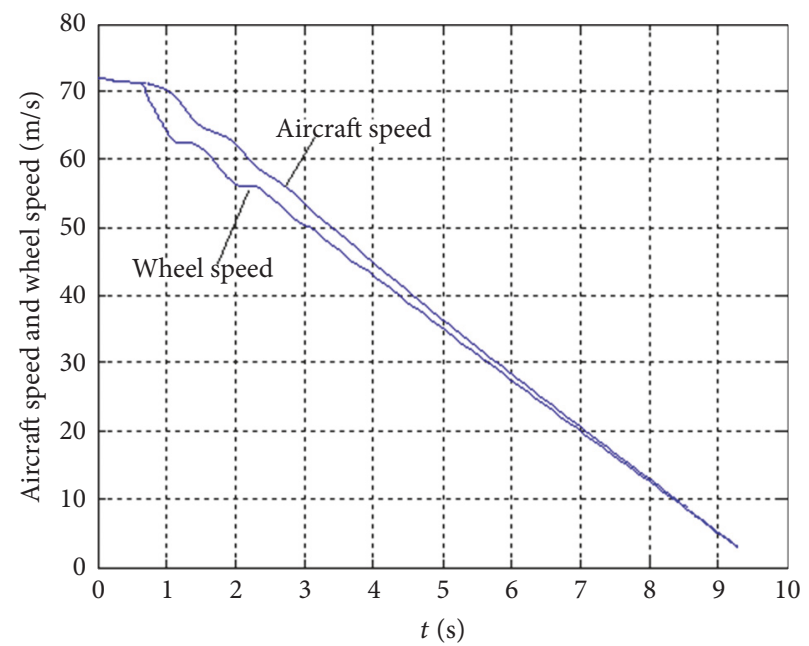

(a) Aircraft speed and wheel speed

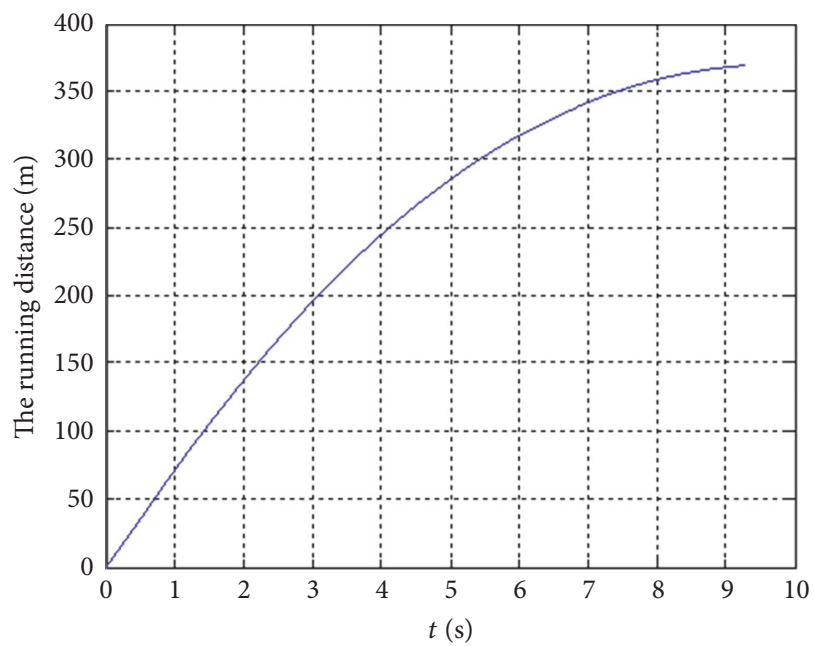

(b) Running distance

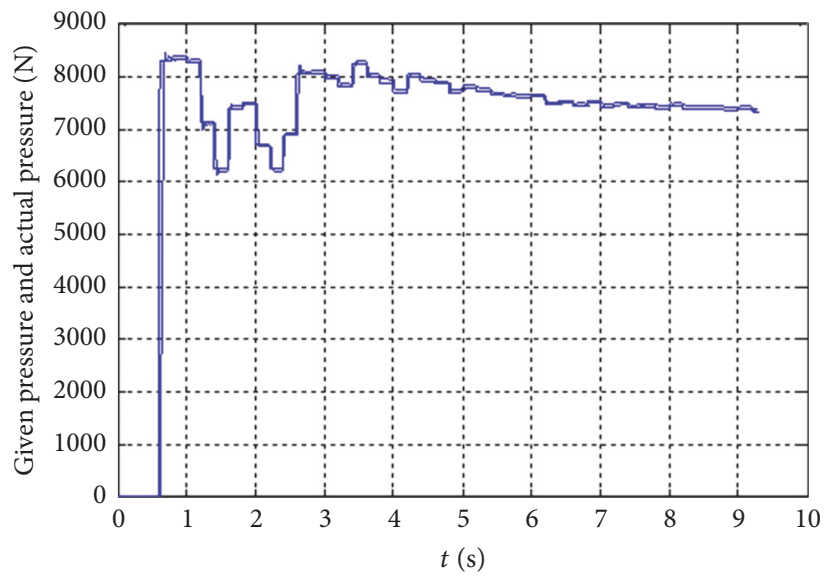

(c) Brake pressure

FIGURE 13: Simulation results in case of emergency brake.

5.3. Analysis of Results. According to the simulation results, the following conclusions can be obtained.

(1) With the increase of the deceleration rate, the brake pressure is bigger, the speed is faster, and the running distance is shorter. In case of the emergency brake, braking force is maximum, so the braking distance is the shortest.

(2) Taking the deceleration rate of 2.1 as an example, the ideal braking time is $72 / 2.1(34.29) \mathrm{s}$, the actual simulation time is about $35 \mathrm{~s}$, and the aircraft speed and wheel speed are in good agreement.

(3) When the brake pressure is simulated in MATLAB environment, the brake pressure is in good agreement with the actual brake pressure. When the deceleration rate is 2.1, brake pressure is about $3000 \mathrm{~N}$.

According to the results of HILS, the following conclusions can be obtained.
(1) With the increase of the deceleration rate, the brake pressure is bigger, the speed is faster, and the running distance is shorter. In case of the emergency brake, braking force is maximum, so the braking distance is the shortest.

(2) Taking the deceleration rate of 2.1 as an example, the HILS time is about $34.95 \mathrm{~s}$, and the aircraft speed and wheel speed are in good agreement. When the deceleration rate is 2.1, brake pressure is also about $3000 \mathrm{~N}$ in HILS.

(3) Since the start of the motor requires a certain reaction time, the actual brake pressure has a certain delay compared with the given pressure in HILS. But from the waveform and size of the HILS results, the actual brake pressure and the given pressure have good consistency.

By comparing the simulation results with the results of HILS, we can know that the simulation results are in good agreement with the HILS results. The results of HILS can reflect the actual working state of the brake system. 


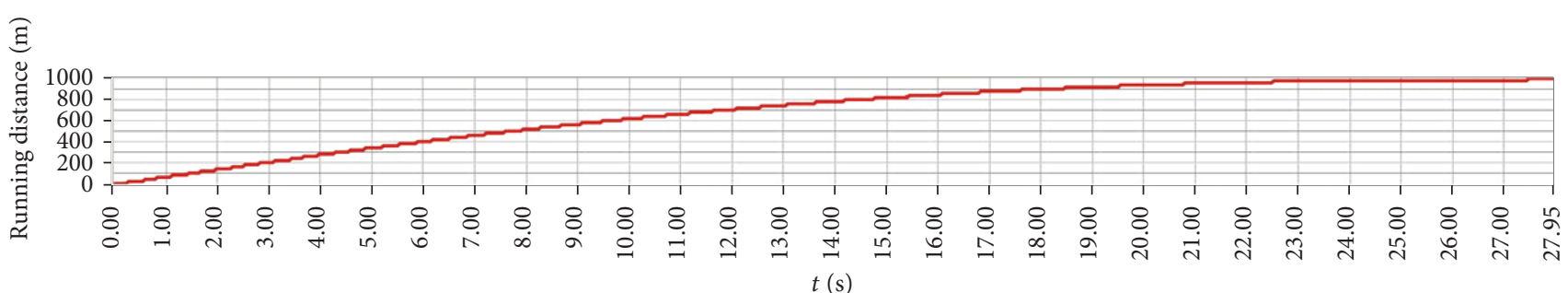

(a) Running distance

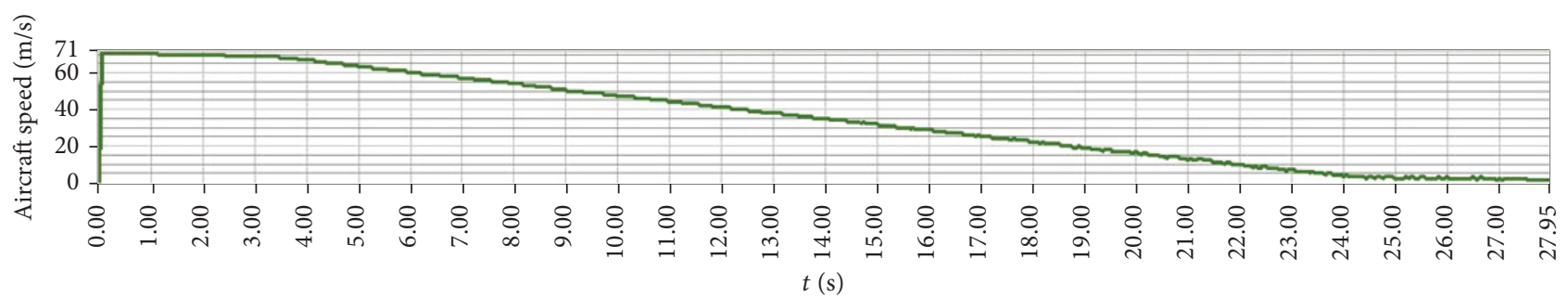

(b) Aircraft speed

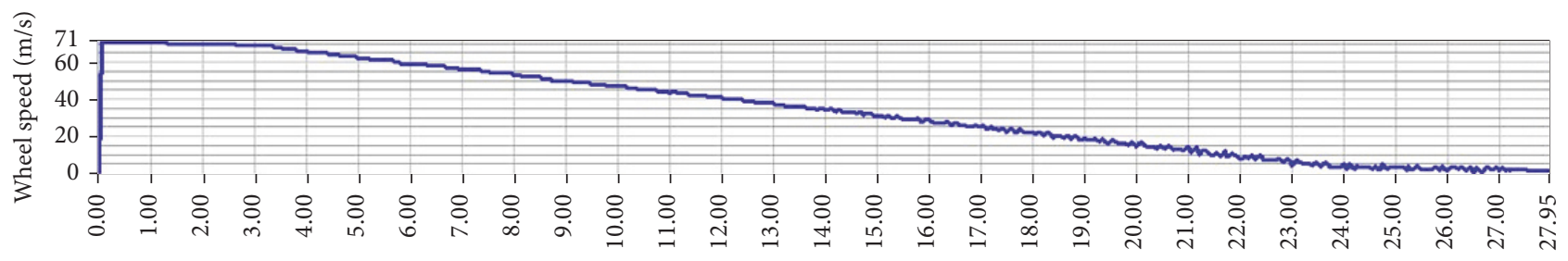

$t(\mathrm{~s})$

(c) Wheel speed

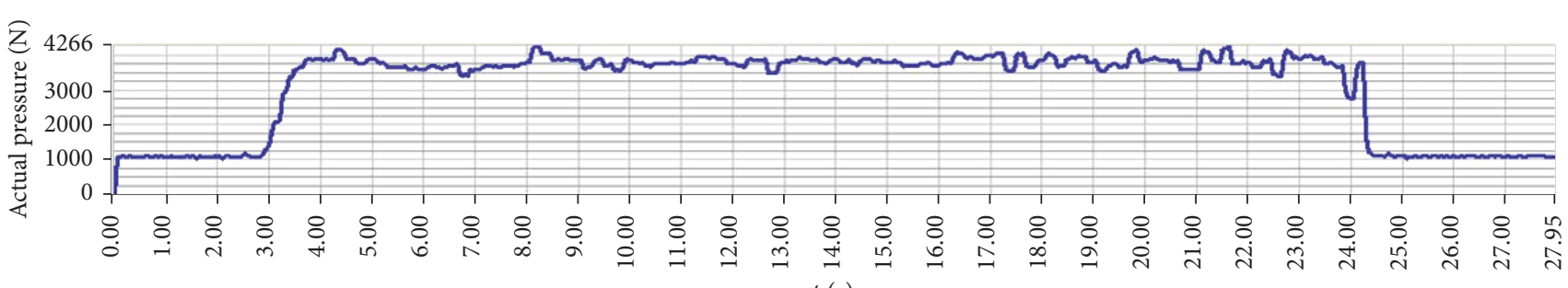

$t(\mathrm{~s})$

(d) Actual brake pressure

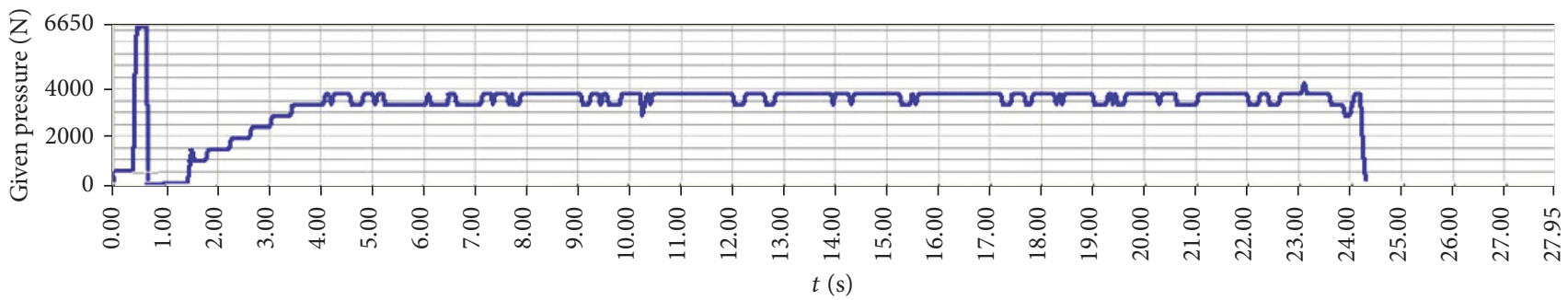

(e) Given brake pressure

FIGURE 14: HILS results when deceleration rate is 3.05 .

\section{Conclusion}

In this paper, the HIL simulation technology is introduced into the aircraft electric braking system. By making full use of the RTW toolbox of MATLAB and DSP, the HIL simulation platform of the aircraft brake system is completed.

The automatic generation of the simulation model code, the online adjustment of the control law parameters in 


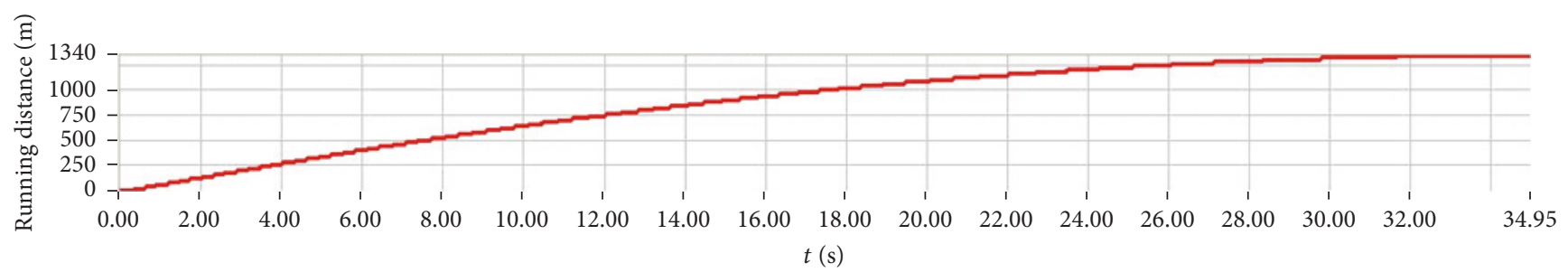

(a) Running distance

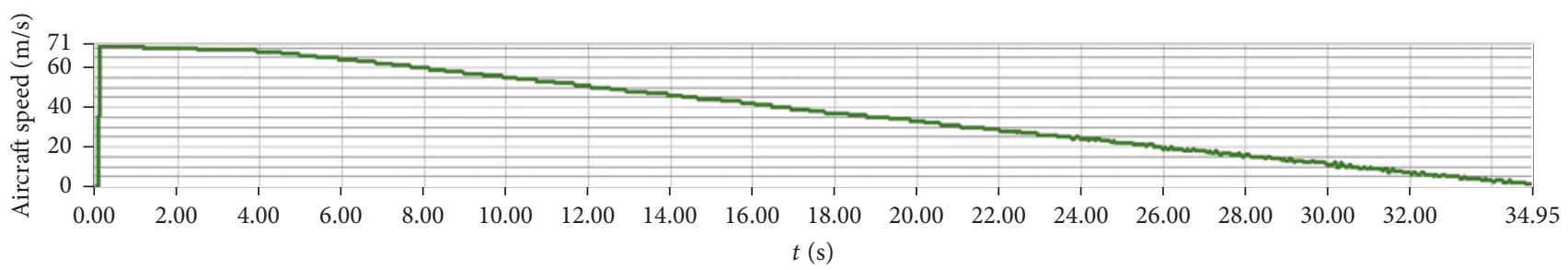

(b) Aircraft speed

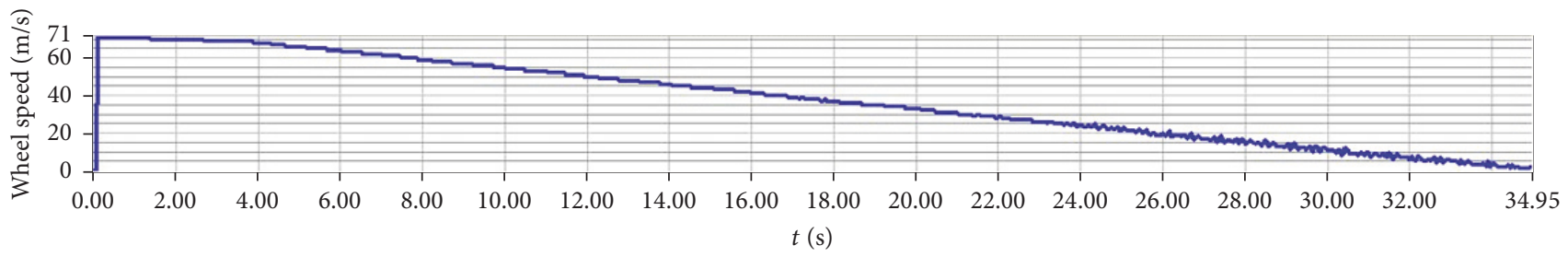

(c) Wheel speed

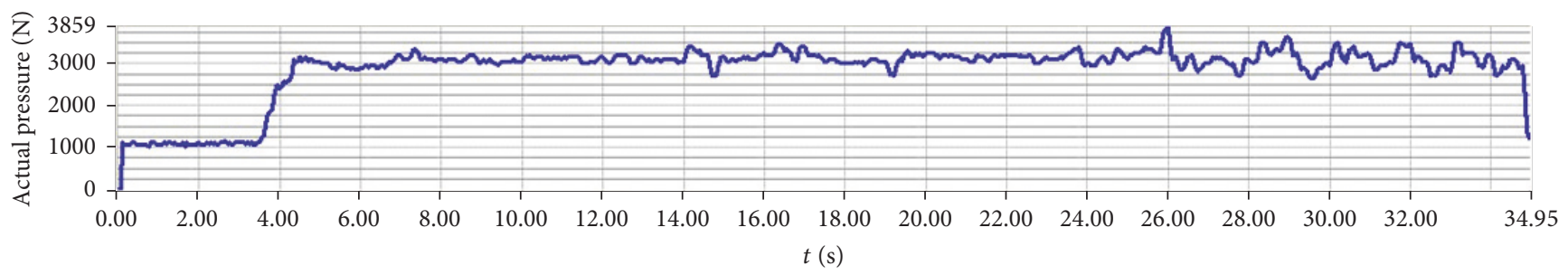

(d) Actual brake pressure

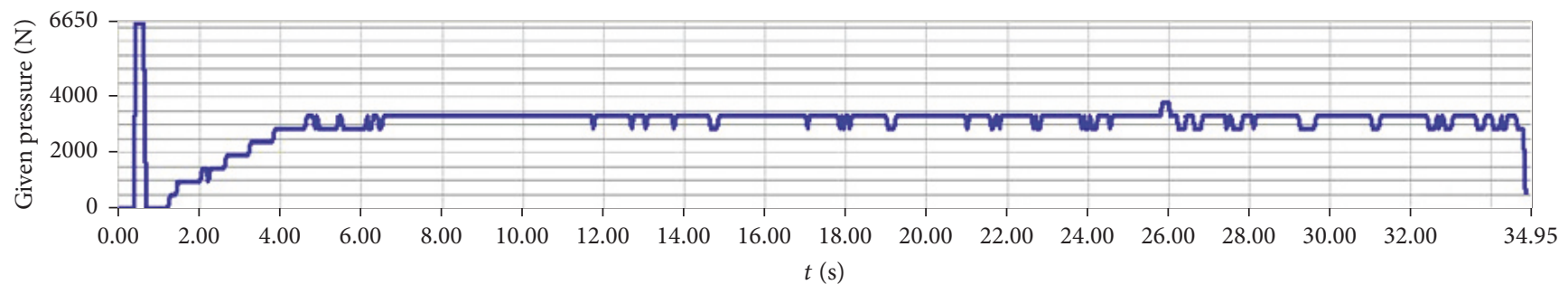

(e) Given brake pressure

FIGURE 15: HILS results when deceleration rate is 2.1.

the simulation process, and the online monitoring of the simulation data in the simulation process are realized.

From the results of simulation and HILS, the simulation curves are very close to the HILS results, which shows that the results in HILS environment are realistic and reliable.

Based on the HILS system, the brake control law can be easily modified or designed. It provides a platform for the design of brake system and the design of control law.

\section{Conflicts of Interest}

The authors declare that there are no conflicts of interest regarding the publication of this paper.

\section{Acknowledgments}

The work is supported by Foundation for Shaanxi Key Laboratory of Small and Special Electrical Machines and 


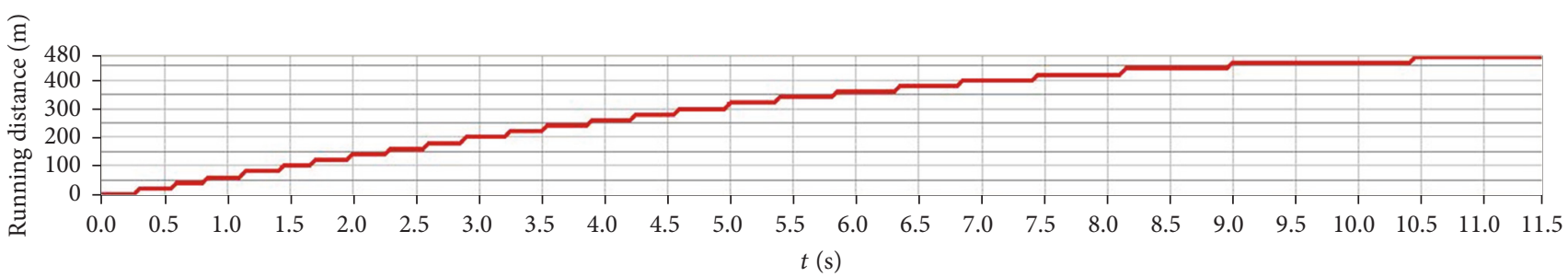

(a) Running distance

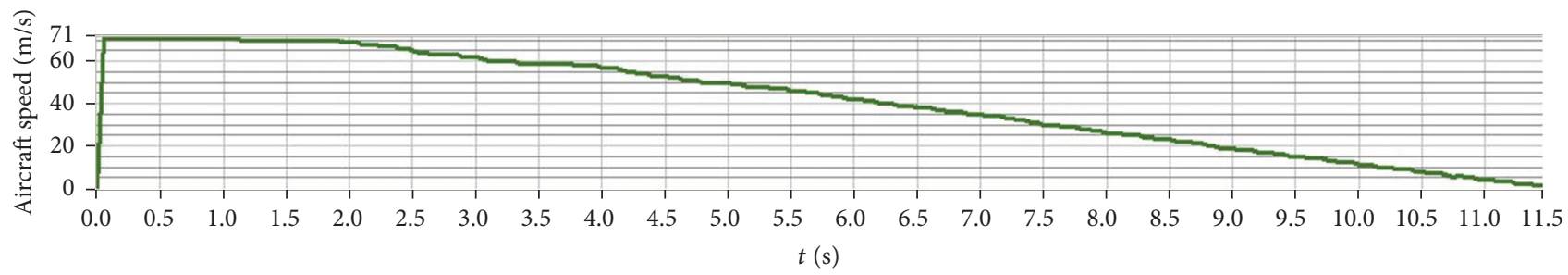

(b) Aircraft speed

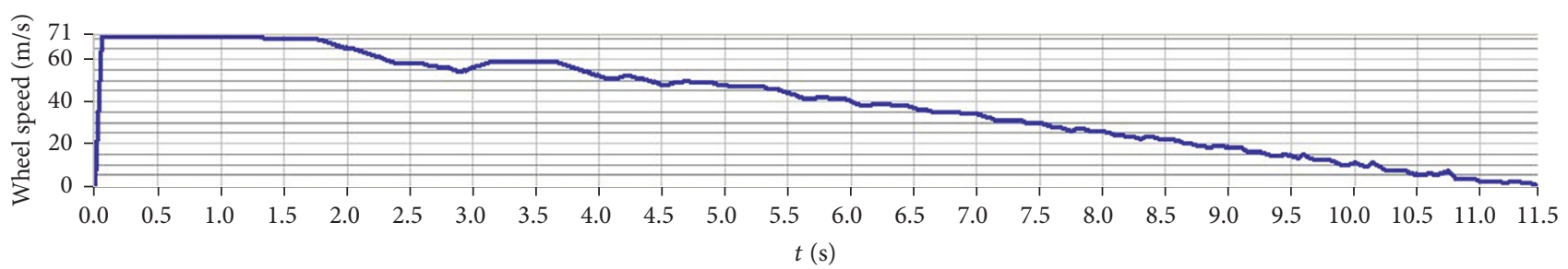

(c) Wheel speed

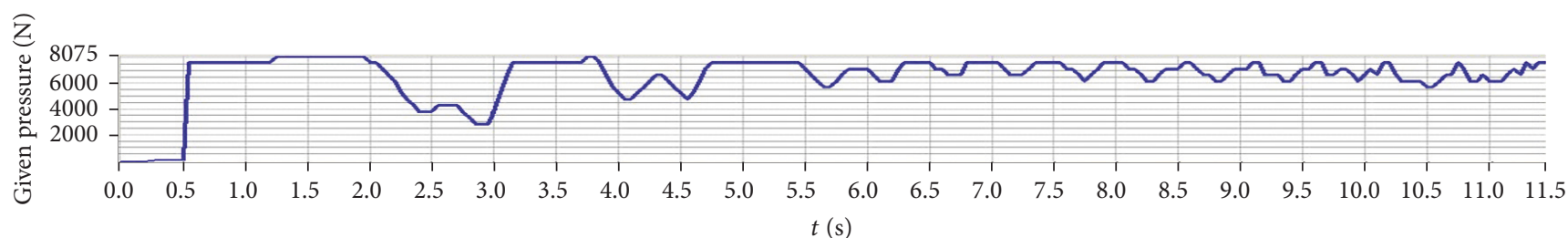

(d) Actual brake pressure

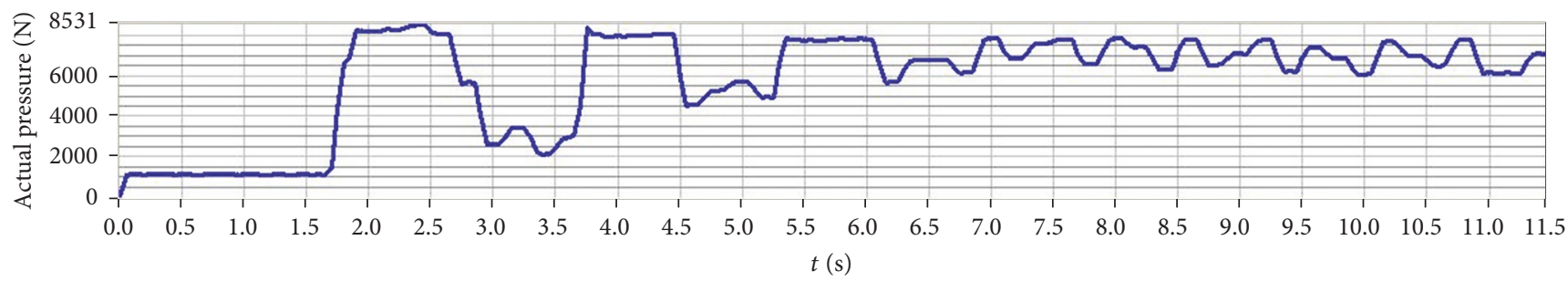

(e) Given brake pressure

FIGURE 16: HILS results in case of emergency brake.

Drive Technology (2014SSJ1004). The work is also supported by National Natural Science Foundation of China (51407143).

\section{References}

[1] J. Wang and L. Xie, "Real-time software design of hardware in-the-loop simulation of aircraft braking system," Computer Measurement \& Control, pp. 773-775, 2009.

[2] M. Yoon, J. Lee, and J. Hong, "The simulink model of motor system for HEV using HILS (hardware-in-the-loop)," in Proceedings of the 2012 Sixth International Conference on Electromagnetic Field Problems and Applications (ICEF), pp. 1-4, Dalian, China, June 2012.

[3] J. W. Jeon, G. A. Woo, K. C. Lee, D. H. Hwang, and Y. J. Kim, "Real-time test of aircraft brake-by-wire system with HILS \& dynamometer system," in Proceedings of the IEEE International Conference on Mechatronics 2004, (ICM '04), pp. 322-327, June 2004.

[4] N. E. Daidzic and J. Shrestha, "Airplane landing performance on contaminated runways in adverse conditions," Journal of Aircraft, vol. 45, no. 6, pp. 2131-2144, 2008. 
[5] H. B. Pacejka and E. Bakker, "The magic formula tyre model," Vehicle System Dynamics, vol. 21, supplement 001, pp. 1-18, 1993.

[6] D. Xu, Y. Li, and L. Xie, "Research on Modeling and Simulation of Aircraft Anti-Skid Braking System," Measurement \& Control Technology, vol. 23, pp. 66-68, 2004.

[7] J. I. Miller and D. Cebon, "A high performance pneumatic braking system for heavy vehicles," Vehicle System Dynamics, vol. 48, no. 1, pp. 373-392, 2010.

[8] H. Zhang, G. Xu, W. Li, and M. Zhou, "Fuzzy logic control in regenerative braking system for electric vehicle," in Proceedings of the 2012 IEEE International Conference on Information and Automation, ICIA 2012, pp. 588-591, June 2012. 


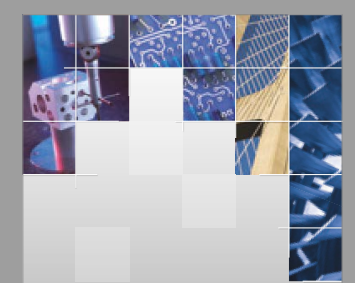

\section{Enfincering}
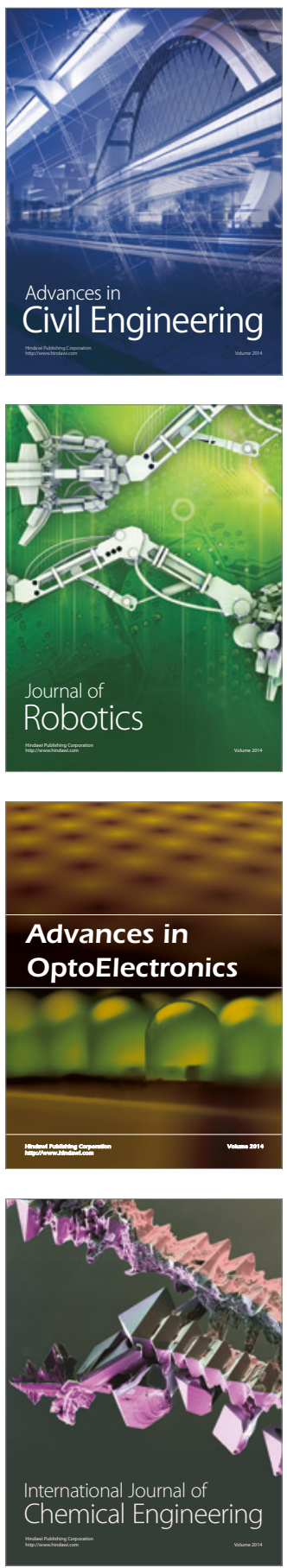

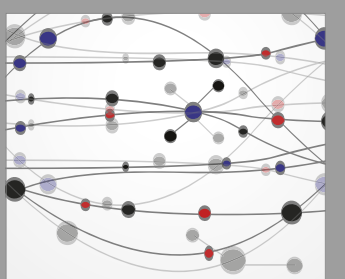

The Scientific World Journal

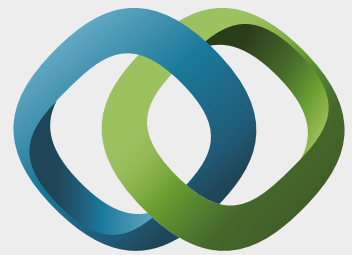

\section{Hindawi}

Submit your manuscripts at

https://www.hindawi.com
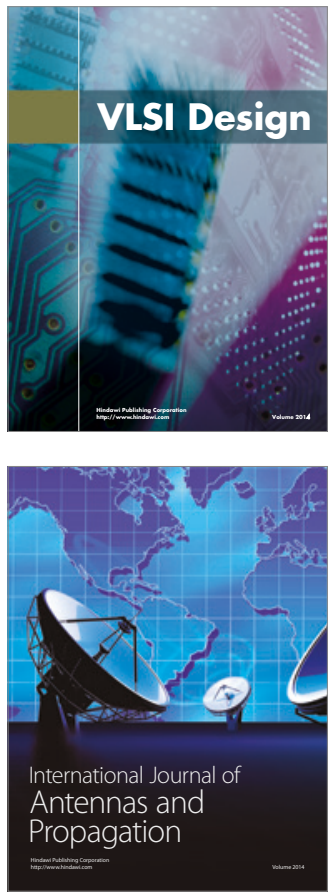

\section{Rotating}

Machinery
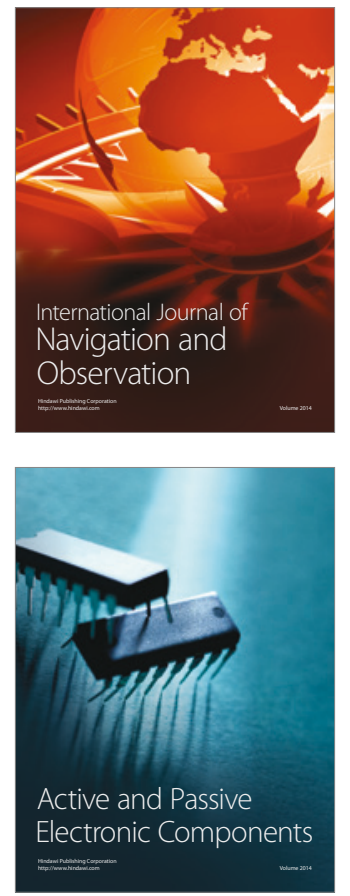
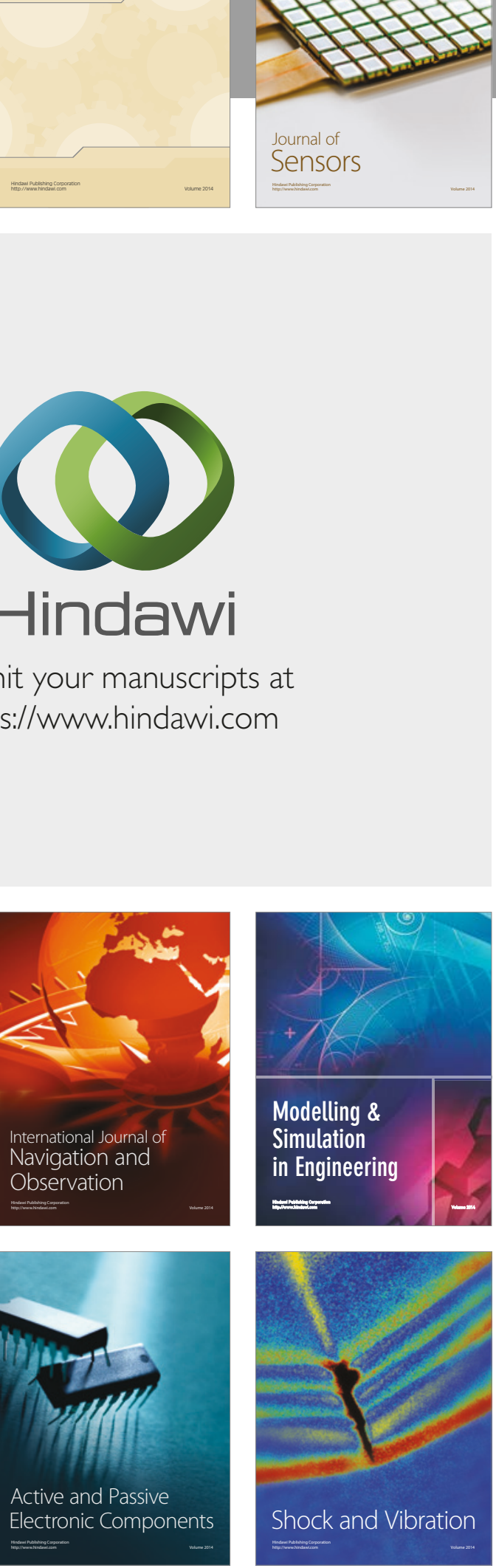
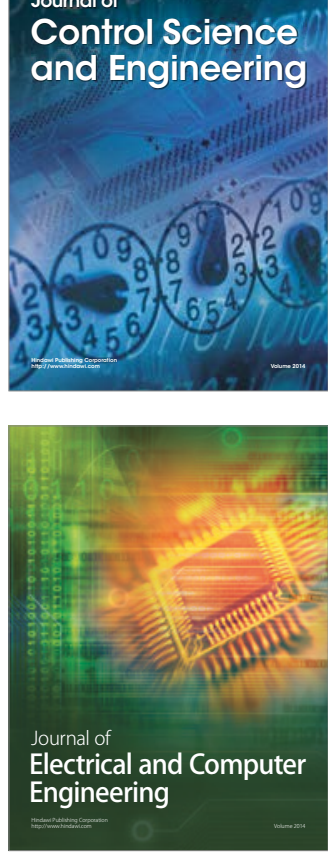

Distributed

Journal of

Control Science

and Engineering
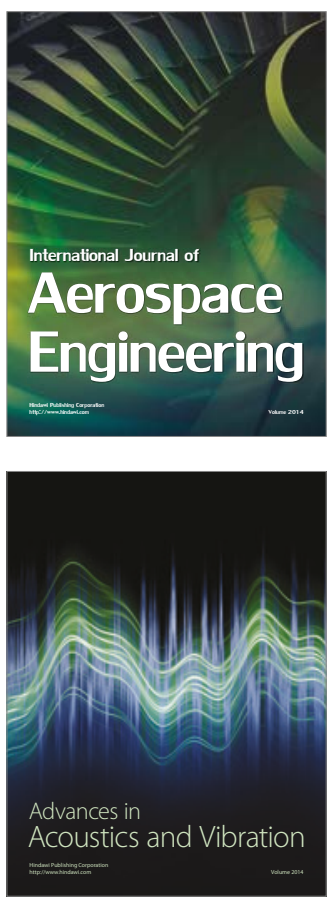

Sensor Networks 